\title{
Approaches to Information Measurement of the Structure State of Technical Systems
}

\author{
Dulesov Aleksander Sergeevich \\ Katanov Khakass State University \\ Abakan, Russian Federation \\ dulesov@khsu.ru \\ Eremeeva Olga Sergeevna \\ Katanov Khakass State University \\ Abakan, Russian Federation \\ yeremeevaos@yandex.ru
}

\author{
Karandeev Denis Jurevich \\ Katanov Khakass State University \\ Abakan, Russian Federation \\ den_dr_house_1991@mail.ru
}

\author{
Dulesova Natalia Valerievna \\ Khakass Technical Institute of Siberian Federal University \\ Abakan, Russian Federation \\ dulesovanv@mail.ru
}

\begin{abstract}
Opportunities of application of the approaches of known scientists to the information measurement are analyzed. The structure of a technical system is proposed as the object of measurement. This structure is characterized by the amount of system elements, possible number and probabilities of its states. Classical mathematical expressions of information theory are presented and the possibilities of their application for determining the information amount and information entropy are established. The issues of accumulation, removal of uncertainty and regulations of the variety of information with reference to considering structures of system are touched.
\end{abstract}

Keywords - information theory, information uncertainty, structural system reliability, system variety, problem of regulation

\section{INTRODUCTION}

Considering a technical system from the standpoint of the presence of information, an important aspect is the understanding of opportunities of its application to the assessment and measurement of a system state. The role of information is a key not only in Informatics [1], but also in system analysis, mathematics [2] and other sciences [3, 4]. In addition, the concept of "information" is poorly formalized because of the vastness, depth and vagueness of information and therefore it has many different definitions, for example, presented in $[5,6]$. Considering the existing set of concepts, their unity and internal properties, the information will be considered as a reflection in time of states of a technical system in a form of a sequence of data that are to be updating. Such determination of information doesn't exclude its receipt, transmission, processing and registration.

Information coming from the technical system indicates its activity. Over time, information allows to enlarge the knowledge through the accumulation, systematization of data, opinions, their interpretation and reinterpretation.

Information about the system state is so varied and voluminous that it doesn't allow holistically to take into account the solution of a wide range of tasks, for example, associated with the operation of a system. The following will be focused only on the issues of obtaining information in relation to the consideration of the structural content of a system. Systems that have a well-defined structure of connections between the elements include network objects: electrical, thermal, water supply, computer networks and similar to them.

\section{STATEMENT OF A PROBLEM OF MEASURING INFORMATION}

Let there be a technical system of a network structure, which can be represented as an undirected graph. Arcs network elements, vertices - the "joints" between the elements. Each arc has indicators that are required to determine the amount of information.

An element can be represented as a discrete device having one input, one output, and it is situated in one of two opposite states at each moment of time. The change of system states is a time-dependent physical process that reflecting the transmitted messages as a time function (when information is presented in the form of primary and constant signals: for example, operable and non-operable states).

The complex system requires control by a person, who requires that any element would transmit a message about its state at certain points in time. Under the impact of the planned solutions and random factors, the element discretely changes its state to the opposite, which is reported to the person. There are such states that can't be fixed for a number of reasons and they will not be taken into account.

Formally, the element can be specified in the form of ordered components of certain sets:

$$
A=(X, Y, P, \delta, N, t)
$$

where $X, Y$ - the final input-output energy flow; $N$ - the set of internal states, $p \in P$ - the set of occurrence probabilities of random events, $\delta$ - the random transition function from one state to another (the opposite). As the function of the 
transitions is predominantly random in nature, then this discrete device is a probabilistic and functioning at discrete instants of time $t$.

During the period under consideration, an energy flow of a discrete type arrives in the input device and the frequency and length of energy flow is changed in the output. This discrete device can be considered as the source of messages that in each moment of time $t$ under the influence of random factors takes one value from a finite set of possible discrete states. Each state of the source $U$ is associated with a symbol in the form of a sign (e.g. "0" or "1"). For each $i$-th state is characterized by a set of combinations of characters $u_{1}, u_{2}, \ldots$, $u_{i}, \ldots, u_{N} u_{-} i \epsilon U$, the number of which is equal to $N$.

Taking into account the existing classical approaches to understanding the role of information and having initial data (a structure, a set of indicators characterizing the states of elements through combination of signs), it is necessary to determine the amount of information inherent in the element, and subsequently the system itself.

\section{ANALYTICAL ASPECTS AND SYNTHESIS OF INFORMATION}

Consider a set of the existing models as applied to the solution of the problem. The conditions to determine the amount of information:

- information is a subject to accumulation;

- only statistical data about the state of the network structure are considered;

- information must be connected with its uncertainty;

- the simplest discrete states are considered;

- the logarithmic measure having additivity is used.

As the nature of the functioning of the element is random, then it is impossible with a high confidence level to assume its further behavior (i.e., its state), thereby having the information uncertainty is available. If to talk about a measure of this uncertainty, then it is considered as a measure of a quantitative information. To analyze the states of the technical system, the measure must satisfy the condition of its monotonous increase. The increase in the number of possible states reaches the amount of $N_{i}$ that characterizes the element $i$ or the discrete device as a source or generator of random events.

In addition, let's note the importance of the additivity property. Let the system consists of $n$ elements, with the amount of states for each of them: $N_{1}, N_{2}, \ldots, N_{i}, \ldots, N_{n}$. All these states belong to the system. Therefore, according to the additivity property, information uncertainty of the system will be equal to the sum of information uncertainties of all elements. Therefore, for a closed system, its information - the sum of information of each of all possible states combinations.

Let's consider further the role of the classical concepts of information and models for its description, with regard to technical systems.

\section{R. FISCHER'S INFORMATION}

Solving the problems of mathematical statistics, R. Fischer considered the general ways of measuring the amount of information, noting the following: the higher the entropy of the source of information, the more information can be obtained from it [7]. Fisher has associated it with the expectation of uncertainty, the value of which is expressed by negative logarithm of the probability of any experiment outcome, thereby touching upon the question of the logarithmic measure of information uncertainty. This measure is used in the analysis of the technical systems $[8,9]$.

\section{R. HARTLEY'S INFORMATION}

He is a founder of information theory and has proposed of a measure of information amount that calculated using the logarithm of this value, observing the principle of additivity [10].

Let' s note the role of the Hartley's model in the problem of obtaining information for the technical system. Knowing the number of elements in the system and the number of its possible opposite states $m=2$, then the number of equally probable states of the entire system is equal to $N=m^{n}$, where $n$ - the number of elements in the system. Then the amount of information $I$ according to Hartley contained in the selected message about a system state is determined as the binary logarithm of $N$ :

$$
I=\log _{2} N
$$

where $N$ - the number of equally probable events; $I-$ the number of bits in the message received on the condition that any of the $N$ events has occurred.

Note that: since the system can be in $m>2$ states, then the base of the logarithm in (1) can be arbitrary, that is $\log _{\mathrm{m}} N$.

Importance in information determination is that the Hartley's binary logarithm is often interpreted as an entropy of a set of equally probable opportunities. In this case, formula (1) is written in the following form:

$$
I=\log _{2} N=\log _{2}(1 / p)=-\log _{2} p
$$

As each of the $N$ states has an equally probable outcome, then $p=1 / N=>N=1 / p$.

Let's consider the Hartley's model for solving the problem of accumulation of information in the operation of a technical system. It is obvious that a complex system has many elements, each can be considered as a subsystem, and its internal content is sometimes unknown for certain analysts. In fact, the system element for the analyst - a "black box", which is considered as a discrete device that generates information about its state in output. As for simultaneous interrelated internal states of the "box", information about them has uncertainty. To determine it, it is necessary to put forward the 
assumption of existence of a specific number of components (elements) in the discrete device. Thus, it is necessary to determine the number of components of the "black box". Depending on their numbers, the amount of information will change in output of the "box". The more one assumes the presence of components, the more information one has. Increasing the number of components, thereby the accuracy requirement of the obtained information in the output of the "box" is increased. The more elements, the higher the number of internal states, due to the complexity of correlations in the system. Therefore, it is necessary to choose only one variant from the diversity of variants about the presence of a number of the box components. In this case, this variant must satisfy the requirements, such as the reliability of the system [11]. After choosing the variant, the generated information becomes known, thereby it passes from the uncertainty category into the category of the known information.

Let's further consider the connection between the obtained information amount and the choice of a variant from a possible finite set:

1) This variant is considered when the "black box" consists of a single component. In this case, it is possible to consider the "box" as a system element, which, as mentioned earlier, can be in one of two equally probable states $[0,1]$. The information generated by this element according to (1) is equal to 1 bit, since the number of states is $N=2$. If to consider such variant of the "box", then one will have a minimum amount of information indicating that the element is extremely simple and, for the fixed time interval, one will expect only the transition from one state to another state (opposite). If states are equally probable $(p=0.5)$, then each state will have a duration is equal to $t=0.5 T$ throughout considered time interval $T$.

2) The following variant - the presence of two components in the "box". To each of the parts, as in the first case, 2 states are assigned. The set equally probable states $N=2^{2}=4[00$, $01,10,11]$. Then according to (1) one will have 2 bits of information. Thus, the element generates information of 2 bit about 4 events with equal probabilities of the joint states $(p$ $=1 / 4=0.25$ ) and the duration of each $t=0,25 T$.

3) For the three components: $N=2^{3}=8[000,001,010$, $011,100,101,110,111] ; I=3$ bits; $p=1 / 8=0,125 ; t=0,125 T$.

The three presented variant indicate that information is a monotonic function of the power of the initial set $N$. The considered flow of events in time is the generation of information and its accumulation. According to the value of this information, it is possible to judge what requirements one can impose for the considered discrete device or system: the level of complexity, a sufficient amount of information, a number of elements, etc.

According to these requirements of determining the information, the Hartley's approach, in a simplified quality, allows to select options (taking into account additivity) according to the principle: the increase of the original length of the number $\left(N_{1}=2\right)$ by $n$ times increases the amount of information to the $n$-th power times $\left(N=2^{n}\right)$. This principle is inherent in the logarithm:

$$
\log _{2} N=\log _{2}\left(N_{1}\right)^{n}=n \cdot \log _{2}\left(N_{1}\right)
$$

When using expressions (2) and (3), then it is possible to measure the amount of information in the case of equally probable events, satisfying the conditions of monotonicity and additivity. However, the Hartley's model has an essential disadvantage: only combinatorial states are taken into account.

\section{K. SHANNON'S INFORMATION}

The Shannon's model reflects a probabilistic approach, expanding the possibilities in determining the amount of information, in contrast to the Hartley's approach [12]. Claude Shannon has proposed a formula for determining the amount of information through different probabilities of the events [13]. In relation to technical systems, the model makes it possible to track the process of increasing information that indicates the increase (accumulation) of knowledge and, consequently, the decreasing of ignorance or information uncertainty.

Let's accommodate a transition from Hartley's model to Shannon's model for revealing the role of this model in the problem of measuring the amount of information of technical systems. The Hartley's formula (1) for non-equiprobable alternatives is a special case of the Shannon's formula having the form:

$$
I=-\sum_{i=1}^{N} p_{i} \log _{2} p_{i}, \text { by } \sum_{i=1}^{N} p_{i}=1
$$

where $I$ - the information amount; $N$ - the number of possible events; $p_{i}$ - the occurrence probability of $i$-th event.

The expression (1) allows to determine a own (private) information about the $i$-th state in the interval $t_{i}$ :

$$
I_{i}=\frac{1}{N} \log _{2} N=\log _{2} N^{\frac{1}{N}}=-\frac{1}{N} \log _{2} \frac{1}{N}
$$

As formula (5) takes into account the equally probable states of the element, then information about the state of the element $i$, according to (2), will be determined as:

$$
I_{i}=p \log _{2} N=\log _{2} N^{p}=-p \log _{2} p
$$

In turn, the complete information about the element state: - in relation to (5), $I=N \cdot I_{i}$ and can be determined by (1); - in relation to (6): 


$$
I=\sum_{i=1}^{N} I_{i}=-\sum_{1}^{N} p \log _{2} p .
$$

The expression (7) obtained on the basis of the Hartley's model coincides with the Shannon's formula (4) for equally probable events. In this case, the information calculated by (7) will indicate the chaotic state of the system and the maximum variation of the structure that is not acceptable for technical systems solving the problems of quality and reliable resource supplying consumers [14]. Shannon's approach for a technical system, according to (4), allows to avoid the considering of equally probable events of the opposite quality ("yes" or "no", "1" or "0") to unequally probable.

The importance of Shannon's formula is the following: if a probability of occurrence of an event is increased, then the amount of information about this event in the message is reduced. Thus, the determined information amount about the object state depends on the probability of this event.

Let's proceed from consideration of the information to the search of the informational entropy. To determine the amount of entropy $H$ for an element, it is necessary (based on the Hartley's model) to determine the number of its components. Applying the Shannon's principle, it is sufficient to determine the probability value $P:\left\{p_{1}, p_{2}, \ldots, p_{i}, \ldots, p_{N}\right\}$ of each of the considered states. To determine a set of these probabilities is possible, using the available statistics, and in its absence the empirical methods [15]. Taking into account the lack of possibility to determine the deterministic content of the expected states of the system, the Shannon's model allows to calculate the information entropy, i.e. the amount of information indicating the presence of uncertainty.

Measurement of information is associated not only with determining the amount of the entropy. Requirements in support of the high level of reliability are imposed to technical systems [16]. There is a widespread use of a such parameter as "time between failures" [17] or operable state [18]. This parameter is measured by the amount of time and its duration and it is much longer than the system failure time. The statistics records both the frequency and duration of failures, and the frequency and duration of the time between failures. Both parameters are caused by the occurrence of events of a probabilistic nature and have a mathematical expectation of the distribution of private information $-\left\{I_{1}, I_{2}, \ldots, I_{i}, \ldots, I_{N}\right\}$.

As the private information, as a random variable, is an average characteristic, then expression (4) allows to determine the amount of information for the two qualitatively different components [19]:

$$
I=-\left(\sum_{i=1}^{N_{1}} p_{i} \log _{2} p_{i}+\sum_{j=1}^{N_{0}} q_{j} \log _{2} q_{j}\right)
$$

$$
\text { by } \sum_{i=1}^{N_{1}} p_{i}+\sum_{j=1}^{N_{0}} q_{j}=1,
$$

where $p_{i}$ and $q_{j}$ - the probability of opposite states (e.g., $p_{i}-$ the probability of a operable element state, $q_{j}$ - the probability of non-operable element state), the number of operable $N_{1}$ and non-operable $N_{0}$ element states.

In fact, the entropy $H$ and information $I$ are determined by the same expression (4), and for two opposite states, according to the expression (8). Consequently, the entropy, as information, can be determined by the Hartley's $\mathrm{f}$ formula: $H=\log _{2}(N)$. It shows that the more states $N$ are considered, the higher entropy $H$ (uncertainty) is. According to Ralph Vinton Lyon Hartley, the number of states $N=N_{1} \cdot N_{0}=2^{n}$ (where $n$ is an integer) forms a set of integers: $\{1,2,4,8,16,32,64 \ldots\}$. Therefore, the entropy will be obtained as an integral number (bit or $n$ components of the "black box"). Regardless of which model will be accepted for calculations, the entropy and information are mathematically and logically related to each other. Therefore, the quantitative value of the entropy, obtained on the basis of assumptions about the probabilistic occurrence of an event, can be compensated by the amount of information when considering this event.

In addition to the mathematical connection between the entropy $H$ and information $I$, there is a correlation. It is associated with necessity to remove the uncertainty in the emergence of adverse situations. In technical systems, the question of removing uncertainty relates to design and operation. Here the determination of entropy is associated with the presence of statistical data on the elements states, and, in case of their lack, with using empirical data. Uncertainty can be partially removed, so the amount of information $I$ is determined by the subtraction of entropies "before" $\left(H_{1}\right)$ and "after" $\left(H_{2}\right)$ of the considering the situation about the system state:

$$
I=H_{1}-H_{2}
$$

In (9), the value of $I$ must be positive that indicates the removal of uncertainty. In practice, the removal of uncertainty is possible, for example by partial replacement of equipment and timely execution of preventive maintenance.

A drawback of the Shannon's model: it is not possible to distinguish states with the same probability, inherent in various elements of the technical system.

\section{N. WIENER'S INFORMATION}

N. Wiener's information relates to the question of the ratio between information and entropy using quantitative values. $\mathrm{He}$ has suggested to use a logarithmic measure as one of the fundamental characteristics of natural phenomena comparable to the amount of substance (material quantity) and the amount of energy [20]. Spreading his idea on the problem of the behavior of the technical system, note the following: in the life 
cycle of the system [21], chaos correlates with orderliness expressed through the amount of information [22]. These two measures don't exclude the application of mathematical statistics and are associated with the concept "amount of information" that introduced by $\mathrm{C}$. Shannon in the practice of calculations [23].

\section{W. ASHBY'S INFORMATION}

W. Ashby has formulated the law of requisite variety, which is based on the ideas of N. Wiener and C. Shannon. The essence of this law for technical systems is as following: To control the state of a system, it is necessary to create a controller that would limit the variety of disturbances aimed to the destruction of a system. In turn, the controller should have such a variety of states that could be useful for the system. In the term "variety", Ashby has shown the following characteristics: the number of different elements of the system; the logarithmic measure of this number on the base 2 [24]. Thus the variety for the element: $R=\log _{2} N$, where $N-$ the number of distinguishable states. If to pass to the variety of system states (provided that their functioning is independent), then its value will be written as $R_{\Sigma}=-\sum_{i} \log _{2} N$, where $i-$ the number of the considered system elements.

The presented expressions don't allow to proceed to the variety in the case when there is the necessity of taking into account the structural and other connections between the elements in the system. In this case, it is necessary to apply a more complex mathematical apparatus for determining of $R$ [25-28].

Considering the issues of the system variety, an important role belongs to the controller. It has the essential function that is to maintain states by blocking the flow of variety from external disturbances. As the controller is a control system, which is endowed with the variety itself. The variety in the controller can reduce the variety from disturbances. In this case, one has to refer to the fact that any law of nature is the result of limiting variety because the world would be completely chaotic without variety limitations [29]. And as the behavior of nature is partially predictable, it is possible to judge the presence of a limitation of the variety of the technical system. For its structure, variety is the presence of dissimilar states that non-recurrent according qualitative contents.

Considering the qualitatively different states of the structure of the system $N_{c}$ and the variety of disturbances $N_{b}$, the amount of variety of the controller: $N_{p}=N_{b} / N_{c}$. This ratio quantitatively reflects the law of requisite variety that is proposed by W. Ashby [30]. In the logarithmic form the law can be written as:

$$
\log N_{p}=\log N_{b} / N_{c}=\log N_{b}-\log N_{c}
$$

If to consider only 2 qualitatively different states of the system structure $N_{c}=2$, then $\log _{2} N_{c}=1$. Turning to the information contents of the system structure, one obtains: $I_{6}=$ $I_{p}+I_{c}$, i.e. the information contents of the external disturbances is the sum of the information contents of the system structure and its controller.

Passing to the problem of regulation, it is necessary to determine a set of essential variables and to select a set of allowed states. The regulation will be insufficient if the controller is considered as a transmitter of variety (information) with limited capabilities (resources). Therefore, one will be limited in the conversion of disturbing variety to a variety of allowed states. The actions of the controller in its simplest form can be shown as two opposite states: 1 - the resource is used (to eliminate effects from external influence); 0 - the resource is not used (since the positive effect obtained from the previous regulatory process is not completed). Such regulator will bring information: $I_{p}=\log _{2} N_{p}=1$, by $N_{p}=2$. From (10) it follows that $\log _{2} N_{6}=\log _{2} N_{p}+\log _{2} N_{c}=2$ that indicates the possibility of considering only two disturbing influences, each of which can be in two states $\left(N_{6}=4\right)$.

\section{CONCLUSION}

One of the important aspects of the operation of the technical system is the consideration of information as a reflection in the time period of its states in the form of a sequence of data are going to be updated. The solution to the problem of measuring information of the system structure state is inextricably linked to approaches:

- R. Fischer's approach about logarithmic measure and the possibilities of its application in the accounting information uncertainty;

- R. Hartley's approach about the measure of the information amount, calculated using the logarithm of this value observing of the principle of additivity when solving the problem of the information accumulation in the operation of the technical system;

- C. Shannon's approach about using of the probabilistic characteristics to determine the amount of information inherent in the system structure at the intersection of states elements;

- N. Wiener's approach about the connection between the measure of the orderliness (expressed by the amount of information) and the measure of chaos (a number of the information entropy);

- W. Ashby's approach on the need to regulate the states of the structure elements aimed at limiting the variety of disturbances that associated with the destruction of the technical system.

The above-mentioned hypothesis (partially supported by previously performed research), proposed assumptions (with brief examples) and the analysis of the role of the models of well-known scientists indicate the potential application of information theory and mathematical modelling in measuring information of the structure state of the technical system. 


\section{ACKNOWLEDGMENT}

This research was supported by the grant "UMNIK" 2018 Program of the Russian Foundation for Assistance to Small Innovative Enterprises in Science and Technology (code № 0040353), and the Russian Foundation for Basic Research (RFBR), project number 18-010-00163.

\section{REFERENCES}

[1] Thomas M. Cover, A. Thomas Joy, "Elements of Information Theory", New Jersey: Wiley and Sons, 2006, 2nd edition. P. 383.

[2] A.N. Kolmogorov, "Three approaches to the quantitative definition of information”, International Journal of Computer Mathematics. 1965, vol. 1, pt. I, pp. 3-11.

[3] P. Shambadal, "Development and Application of the Entropy Concept", Nauka, Moscow, 1967. P. 280.

[4] V.A. Alexandrov, A.V. Boyarshinov and P.Z. Wilinzon, "Technics and technology in XXI century: modern state and prospects of development", Novosibirsk: CRNS Of Sibprint, 2009. P. 160.

[5] D.S. Chernavskii, "Synergetics and information", M.: Science, 2001. P. 244.

[6] V.M. Kaziev, "Introduction to analysis, synthesis and modeling of systems", Internet-University of Information Technology; BINOM. Laboratory of knowledge, 2006. P. 244.

[7] B.R. Frieden, "Science from Fisher Information: A Unification", Cambridge University. 2004. P. 504.

[8] A.S. Dulesov, D.Y. Karandeev and N.V. Dulesova, "Reliability analysis of distribution network of mining enterprises electrical power supply based on measure of information uncertainty", IOP Conference Series: Earth and Environmental Science (EES). 2017, vol. 87. pp. 1-6.

[9] N.A. Kuznetsov, "Information interaction in technical and living systems", Information processes. 2001. vol. 1. pt. 1. pp. 1-9.

[10] R.V.L. Hartley, "Transmission of Information", Bell System Technical Journal., vol. 7, pt. III., 1928, pp. 535-563.

[11] Reda Farag, Haldar Achintya, "A novel reliability evaluation method for large engineering systems", Ain Shams Engineering Journal, 2016, pp. 1-13.

[12] C.E. Shannon, "Mathematical Theory of Communication", Bell System Tech. J., vol. 27, 1948, pp. 379-423.

[13] C.E. Shannon, "Communication Theory of Secrecy Systems", Bell System Tech. J., vol. 28, 1949, pp. 656-715.

[14] A.S. Dulesov and D.Y. Karandeev, "Construction of optimal structure of technical system using "branch and bound", by criteria of profitability and reliability", Journal Safety \& Reliability of Power Industry., vol. 33, 2016, pp. 56-59.

[15] A.S. Dulesov and M.Y. Semyonova, "Subject probability in measure detection of object state uncertainty", Fundamental research. 2012, vol. 3. pp. 81-86.
[16] A.S. Dulesov, D.Y. Karandeev and N.V. Dulesova, "Optimal redundancy of radial distribution networks by criteria of reliability and information uncertainty", 3nd International Conference on Industrial Engineering, Applications and Manufacturing (ICIEAM), 2017. pp. 1-4. DOI: 10.1109/ICIEAM.2017.8076467

[17] Charles E. Ebeling, "An Introduction to Reliability and Maintainability Engineering”, McGraw-Hill Companies, Inc., Boston, 1997, p. 486.

[18] Patrick D.T. O'Connor, "Practical Reliability Engineering", John Wiley \& Sons, 1998, 4th ed. P. 431.

[19] A.S. Dulesov, N.V. Dulesova and D.Y. Karandeev, "Delimitation indicator of level of reliability of technical system on the qualitative character: entropy approach", Fundamental research, vol. 2, pt. III., 2016, pp. 477-481.

[20] N. Wiener, "Cybernetics: Or Control and Communication in the Animal and the Machine", Paris, (Hermann \& Cie) \& Camb. Mass. (MIT Press). 1961, 2nd revised ed. P. 232.

[21] H. Haken, "Synergetics: Introduction and Advanced Topics", Springer. 1983.

[22] E.H. Liiv, "Infodinamika. Infodynamics. Generalized entropy and negentropy", Tallinn:AO Juhisjelu, 1998, P. 200.

[23] A. S. Dulesov, D. Y. Karandeev and T. G. Krasnova, "The evaluation of the correlation between entropy and negentropy in the structure of a technical system", MATEC Web Conf. International Conference on Modern Trends in Manufacturing Technologies and Equipment (ICMTMTE 2017), 2017, vol. 129, pp. 1-4. DOI: https://doi.org/10.1051/matecconf/201712903005

[24] W. Ross Ashby, "Principles of the Self-Organizing Dynamic System", In: Journal of General Psychology (1947). vol. 37, pp. 125-128.

[25] D.Y. Karandeev, "Accounting the information amount in the task of constructing the optimal structures of the distribution networks with redundancy", Vestnik of Katanov Khakass State University. 2016, vol. 20, 16-19. (in Russian, abstr. in English).

[26] A.S. Dulesov, D.Y. Karandeev and N.N. Kondrat, "Definition of amount of information entropy in structure of the technical system by method of the minimum ways", Journal «Modern high technologies». 2016, vol. 2, pt. III., 425-429. (in Russian, abstr. in English).

[27] A.S. Dulesov, D.Y. Karandeev and N.N. Kondrat "Definition of amount of information entropy in structure of the technical system by method of the minimum sections", Journal «Fundamental research». 2016, vol. 3, pt. III., 472-476. (in Russian, abstr. in English).

[28] A.S. Dulesov, D.Y. Karandeev and N.N. Kondrat "How to define amount of information for the simplest structure of system", Vestnik of Katanov Khakass State University. 2016, vol. 15, 7-9. (in Russian, abstr. in English).

[29] Edward Ott "Chaos in Dynamical Systems", Cambridge University Press. 2002. P. 492

[30] W. Ross Ashby "Introduction to Cybernetics", Chapman \& Hall, 1956, P. 295. 